\title{
Gastric Fistula, CTCAE
}

National Cancer Institute

\section{Source}

National Cancer Institute. Gastric Fistula, CT CAE. NCI Thesaurus. Code C143499.

A disorder characterized by an abnormal communication between the stomach and another organ or anatomic site. 